IPM/P-2010/009

arXiv:1003.4089

\title{
O-BTZ: Orientifolded BTZ Black Hole
}

\author{
F. Loran \\ Department of Physics, Isfahan University of Technology, Isfahan 84156-83111, Iran * \\ M. M. Sheikh-Jabbari \\ ${ }^{2}$ School of Physics, Institute for research in fundamental sciences (IPM), P.O.Box 19395-5531, Tehran, Iran
}

( $\Omega$ Dated: October 30, 2018)

\begin{abstract}
Banados-Teitelboim-Zanelli (BTZ) black holes are constructed by orbifolding $\mathrm{AdS}_{3}$ geometry by boost transformations of its $O(2,2)$ isometry group. Here we construct a new class of solutions to $\mathrm{AdS}_{3}$ Einstein gravity, orientifolded BTZ or $O-B T Z$ for short, which in general, besides the usual BTZ orbifolding, involve orbifolding (orientifolding) by a $\mathbb{Z}_{2}$ part of $O(2,2)$ isometry group. This $\mathbb{Z}_{2}$ is chosen such that it changes the orientation on $\mathrm{AdS}_{3}$ while keeping the orientation on its $2 D$ conformal boundary. O-BTZ solutions exhaust all un-oriented $\mathrm{AdS}_{3}$ black hole solutions, as BTZ black holes constitute all oriented $\mathrm{AdS}_{3}$ black holes. O-BTZ, similarly to BTZ black holes, are stationary, axisymmetric asymptotically $\mathrm{AdS}_{3}$ geometries with two asymptotic charges, mass and angular momentum.
\end{abstract}

PACS numbers: 11.25.Tq, 04.70.-s, 04.20.Dw, 04.60.Rt

\section{Introduction and Summary of results}

Although does not have propagating gravitons, $\mathrm{AdS}_{3}$ Einstein gravity has nontrivial black hole solutions, the BTZ black holes [1, 2], and hence provides a simple but at the same time rich arena for addressing questions about black holes and quantum gravity in general. Since in three dimensions the number of independent components of the Ricci tensor is the same as that of Riemann curvature, all of the solutions to the equations of motion for pure $\mathrm{AdS}_{3}$ Einstein gravity are locally $\mathrm{AdS}_{3}$ and are obtained as quotients of global $\mathrm{AdS}_{3}$ by (a subgroup of) its $S O(2,2)$ isometry [2]. For a generic BTZ black hole solution, however, the quotient leads to closed time-like curves (CTC's) in some regions of the space, which happen to fall behind the inner horizon of the black hole geometry. In order to remove the usual problems with the CTC's, those regions in the BTZ geometry are cut out of the global $\mathrm{AdS}_{3}$. As was discussed in [2] and we review below, the excised region includes a part of the causal boundary of global $\mathrm{AdS}_{3}$ and renders the BTZ black holes as geodesically incomplete.

Besides the geodesic incompleteness, the BTZ solutions also suffer from a quantum instability. To see the instability one may introduce a free scalar theory in the classical BTZ background geometry. It has been shown that [3], see also [4], expectation value of components of the energy momentum tensor of quantum fluctuations of this field blows up at the inner horizon, signaling an instability at the inner horizon, which is also a Cauchy horizon in the (non-extremal) rotating BTZ black hole. This problem seems to be a generic property of BTZ black holes and independent of the details of the quantum field theory in question. Noting that this instability is originating from inside the inner horizon region [3], if one can cut this region in a consistent manner, the problem with the instability, as well as the geodesic incompleteness issue, might be resolved. However, so far it is not clear how the latter should be performed.

It has been argued that the $\mathrm{AdS}_{3} / \mathrm{CFT}_{2}$ provides us with the tools to probe the region inside the horizon 7 . Computations with the $\mathrm{CFT}_{2}$ again reveals the same instability at the inner horizon of the BTZ black hole [8]. The fact that $\mathrm{CFT}_{2}$ correlators blow up at the inner (Cauchy) horizon or at the (orbifold) singularity of the static BTZ has been interpreted as impossibility of probing the region beyond the Cauchy horizon and hence a manifestation of the cosmic censorship conjecture [8].

On the other hand, in the case of pure Lorentzian $\mathrm{AdS}_{3}$ Einstein gravity, which is the case of our interest in this paper, we may have the advantage of a dual $2 D$ conformal field theory $\left(\mathrm{CFT}_{2}\right)$ description [5]. This dual $\mathrm{CFT}_{2}$, if it exists, resides on the conformal (causal) boundary of $\mathrm{AdS}_{3}$, which is a $1+1 D$ flat space. The states of this $\mathrm{CFT}_{2}$ are labeled by representations of a Virasoro algebra with equal left and right central charges $c_{L}=c_{R}=c$. In the seminal work [6] Brown and Henneaux showed that the Virasoro algebra corresponding to the (proposed) dual $\mathrm{CFT}_{2}$ is directly related to a subset of $3 D$ diffeomorphisms respecting certain boundary conditions, and

$$
c=\frac{3 \ell}{2 G},
$$

where $\ell$ is $\mathrm{AdS}_{3}$ radius and $G$ is the $3 D$ Newton constant.

The Brown-Henneaux boundary conditions [6] allow for diffemorphisms which change the orientation on the $\mathrm{AdS}_{3}$ while preserving the orientation on the conformal boundary as well as diffeomorphisms which preserve $\mathrm{AdS}_{3}$ orientation but change the orientation on the 
boundary. The latter set of diffeomorphisms are fixed by the choice of sign for energy and angular momentum in the dual $2 D$ CFT and hence could be discarded. The former, however, may be associated with a well-defined operator in the dual CFT. In this Letter we will focus on such diffeomorphisms.

Motivated by the puzzles (features) of the usual BTZ solutions discussed above, we construct the new class of stationary and axisymmetric "O-BTZ" solutions the line element of which are the same as BTZ everywhere, with the same mass and angular momentum. O-BTZ black holes are obtained by orbifolding or "orientifolding" the orientation changing $\mathbb{Z}_{2}$ part of the $O(2,2)$ isometry of $\mathrm{AdS}_{3}$. This $\mathbb{Z}_{2}$ preserves the orientation on the $2 \mathrm{D}$ boundary of the $\mathrm{AdS}_{3}$ and has a fixed locus which is a space-like cylinder located in the region between the two horizons of BTZ, the "Space-like Orientifold Cylinder" or SOC for short. As such our O-BTZ black holes completes the results of [1] : All possible black hole solutions to $\mathrm{AdS}_{3}$ Einstein gravity are either BTZ or O-BTZ.

From the usual BTZ geometry viewpoint which serves as the covering space for the O-BTZ, hence, this $\mathbb{Z}_{2}$ exchanges the region outside the outer horizon and the region inside the inner horizon. Technically, the "orientifold" $\mathbb{Z}_{2}$ projection in the covering space may be implemented by cutting a BTZ geometry exactly at the middle of the region between its two horizons (where the fixed locus of the orientifold is located) and gluing another copy of the same geometry to it. The O-BTZ geometry is hence the part of BTZ geometry confined between the two orientifold fixed locus which should be viewed as the surfaces at the end of the geometry. As mentioned the O-BTZ everywhere except at the fixed locus of the orientifold projection has the same metric as an ordinary BTZ. O-BTZ geometry in the covering space may be viewed as a solution to $\mathrm{AdS}_{3}$ Einstein gravity with a $\delta$-function source at the fixed locus of the orientifold projection, the SOC.

In this way we obtain a solution which, despite of having the metric of a rotating BTZ (outside its horizon), does not have an inner horizon region. Therefore, these solutions do not have the CTC issue (unlike the standard BTZ geometry). ${ }^{1}$ The analysis of [3] can be repeated for our geometry leading to the result that energy momentum tensor for the fluctuations of any given field on the O-BTZ backgrounds remain finite everywhere. The rest of this Letter is organized as follows. We first revisit

\footnotetext{
1 The idea of cutting the region with CTC's and gluing another part to the geometry for removing the CTC problem has been discussed, e.g. see [9]. Note, however, that our construction, although techincally and in the covering space of the orientifolding seems similar, has the crucial difference that the geometry on the two sides of the "fixed locus" are related by orinetifolding and have exactly the same line element.
}

and review some facts about BTZ black holes. We then introduce the O-BTZ solutions and study their causal structure and some other of their features. We end with discussions and outlook.

\section{BTZ black holes revisited}

$\mathrm{AdS}_{3}$ space is a hyperboloid embedded in $R^{2+2}$ :

$$
-T_{1}^{2}-T_{2}^{2}+X_{1}^{2}+X_{2}^{2}=-\ell^{2} .
$$

A suitable coordinate for $\mathrm{AdS}_{3}$ is [3]

$$
d s^{2}=-\left(\tilde{r}^{2}-\ell^{2}\right) d t^{2}+\ell^{2} \frac{d \tilde{r}^{2}}{\tilde{r}^{2}-\ell^{2}}+\tilde{r}^{2} d \phi^{2}
$$

where $t, \phi \in(-\infty,+\infty)$. For global $\mathrm{AdS}_{3}$ this coordinate system should be extended past $\tilde{r}^{2} \geq 0$. This may be achieved by replacing $\tilde{r}$ with $\tilde{\rho}, \tilde{\rho}^{2}=\ell^{2}-\tilde{r}^{2}$, where $\tilde{r}^{2}$ becomes negative. In the $\tilde{r}^{2} \geq \ell^{2}$ region (region I) $t$ is time-like and $\phi$ is space-like. In the region II, where $\tilde{r}$ and $\tilde{\rho}$ coordinate systems overlap and $0<\tilde{r}^{2}<\ell^{2}, t$ and $\phi$ are both space-like. In region III, where $\tilde{\rho}^{2}>\ell^{2}, t$ coordinate becomes space-like while $\phi$ is time-like. Relaxing $\tilde{r}^{2}>0$ condition, $\tilde{r} \leftrightarrow \tilde{\rho}, t \leftrightarrow \phi$ coordinate transformation is a $\mathbb{Z}_{2}$ diffeomorphism which exchanges regions I and III and maps region II to itself. In the embedding $R^{2+2}$ space this $\mathbb{Z}_{2}$ may be realized as $X_{1} \longleftrightarrow X_{2}, \quad T_{1} \longleftrightarrow T_{2}$. This $\mathbb{Z}_{2}$ changes the orientation on the boundary while preserving the $\mathrm{AdS}_{3}$ orientation. Later we will introduce another $\mathbb{Z}_{2}$, the orientifold $\mathbb{Z}_{2}$, which changes the orientation on $\mathrm{AdS}_{3}$ while keeping the orientation on the $2 D$ boundary.

BTZ black holes [1, 2] constitute all classical solutions to the pure $\mathrm{AdS}_{3}$ Einstein gravity (modulo the self-dual orbifold [10]) with a fixed $A d S_{3}$ orientation and are obtained by orbifolding the original $\mathrm{AdS}_{3}$ by the boosts of its $S O(2,2)$ isometry:

$$
\begin{aligned}
& T_{1} \pm X_{1} \equiv e^{ \pm \frac{2 \pi r_{+}}{\ell}}\left(T_{1} \pm X_{1}\right), \\
& T_{2} \pm X_{2} \equiv e^{ \pm \frac{2 \pi r_{-}}{\ell}}\left(T_{2} \pm X_{2}\right) .
\end{aligned}
$$

Without loss of generality we assume $r_{+}>r_{-} \geq 0$. (For $r_{+}=r_{-}$(4) does not lead to a black hole [2].) For $r_{-}=0$, the static BTZ black hole, the above orbifolding has a fixed line at $T_{1}=X_{1}=0, T_{2}^{2}-X_{2}^{2}=\ell^{2}$ while for generic $r_{-} \neq 0$ case the orbifolding is freely acting on $\mathrm{AdS}_{3}$ and we have a smooth geometry. In the coordinate system (3) the BTZ identification (4) is written as

$$
(t, \tilde{r}, \phi) \sim\left(t-2 \pi r_{-} / \ell, \tilde{r}, \phi+2 \pi r_{+} / \ell\right) .
$$

In the BTZ coordinates the metric takes the form

$$
d s^{2}=\rho^{2} d \tau^{2}+\frac{r^{2} d r^{2}}{16 G^{2} J^{2}-\frac{r^{2} \rho^{2}}{\ell^{2}}}+r^{2} d \varphi^{2}-8 G \ell J d \tau d \varphi,
$$

where $\varphi \in[0,2 \pi]$,

$$
\rho^{2}=8 G M \ell^{2}-r^{2},
$$




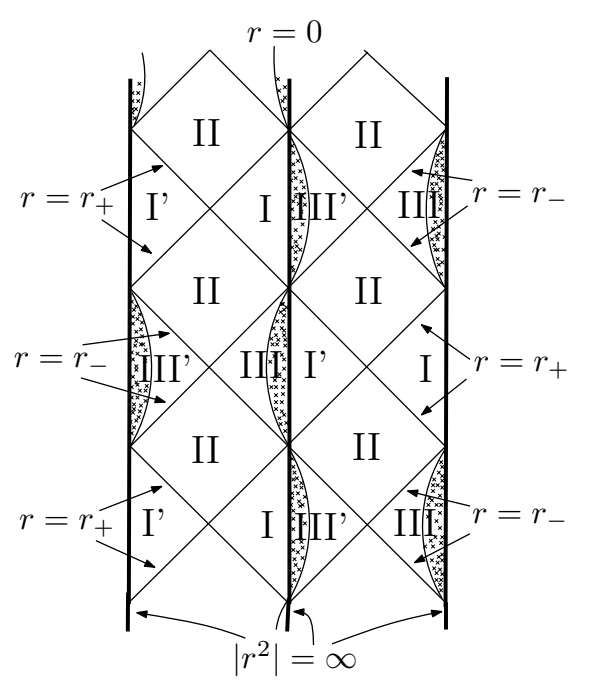

FIG. 1: Two Penrose diagrams of a generic BTZ black hole drawn side-by-side. Regions I, I' are bounded between the boundary at $r^{2}=+\infty$ and the outer horizon at $r=r_{+}$. Region II is the region between the two horizons and Regions III, III' are bounded between the inner horizon and boundary at $\rho^{2}=\infty$. In the BTZ geometry the hatched area, corresponding to $r^{2}<0$ is cut from the BTZ geometry. As depicted, this region contains a part of the causal boundary of original $\mathrm{AdS}_{3}$ [2]. In the figure the $\varphi$ direction has been suppressed and the $\left|r^{2}\right|=\infty$ lines correspond to $\varphi=0, \pi, 2 \pi$. To convey the idea that $r$ coordinate can be extended past $r^{2}<0$ we have drawn two Penrose diagrams side-by-side.

and $M, J$ are (ADM) mass and angular momentum

$$
M=\frac{r_{+}^{2}+r_{-}^{2}}{8 \ell^{2} G}, \quad J=\frac{r_{+} r_{-}}{4 G \ell} .
$$

The BTZ coordinate system (6) has the advantage that the identification (44) which constructs the BTZ solution is performed only on the $\varphi$ coordinate, $\varphi \equiv \varphi+2 \pi$.

From identification (5) it is clear that there are no closed time-like curves (CTC's) in the region where $t$ is parameterizing time. This is, however, not sufficient and with the above identification in the $r^{2}<0\left(\rho^{2}>8 G M \ell^{2}\right)$ region, which is inside the inner horizon of the BTZ black hole, geometry develops CTC's. To remove inconsistencies arising from CTC's one is forced to cut the geometry at $r=0$ 2]. Penrose diagram of the BTZ geometry is depicted in Fig 1

\section{Orientifoleded-BTZ (O-BTZ) solutions}

As mentioned, the only possible classical solutions to $\mathrm{AdS}_{3}$ Einstein gravity should necessarily be locally $\mathrm{AdS}_{3}$ and they are hence all classified by orbifolding $\mathrm{AdS}_{3}$ by its isometries; which if we also demand preserving the orientation on $\mathrm{AdS}_{3}$, that is orbifolding with a subgroup of $S O(2,2)$. These are BTZ solutions [1, 2] or the selfdual $\mathrm{AdS}_{3}$ orbifold [10].

The only remaining possibility which we will study here is then to orbifold (orientifold) $\mathrm{AdS}_{3}$ by the orientation changing $\mathbb{Z}_{2}$ which is a part of $O(2,2)$ but not

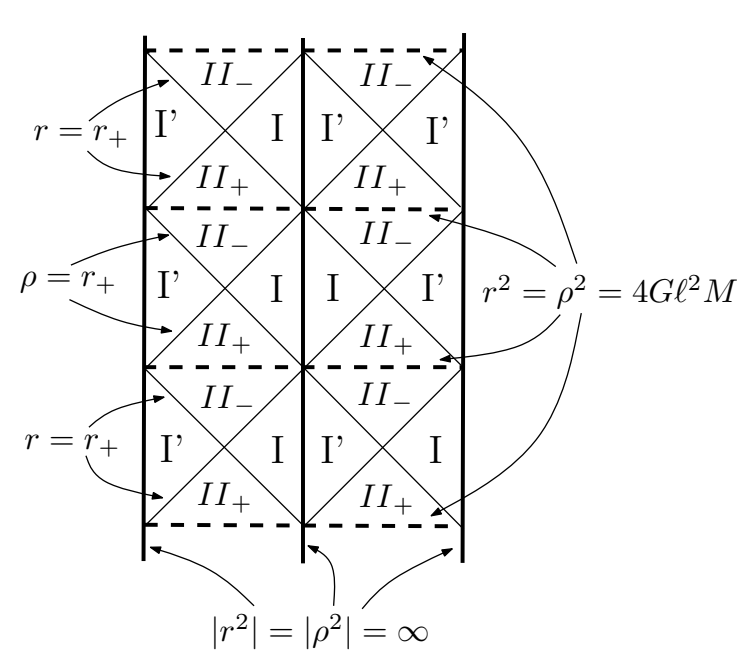

FIG. 2: Two Penrose diagrams of an O-BTZ black hole in the "orinetifold covering space", drawn side-by-side. $\left|r^{2}\right|=\infty$ line is the causal boundary which is a cylinder. Fixed locus of the orientifold projection, the "Space-like Orientifold Cylinder" (SOC), is indicated by the dashed line at $r^{2}=\rho^{2}=$ $4 G \ell^{2} M$. As obtained by the $\mathbb{Z}_{2}$ projection, the regions above and below the horizontal dashed line have the same metric. The O-BTZ geometry is the part which is limited between two successive dashed lines. As it is seen the O-BTZ geometry, is geodesically complete and does not have the inner horizon or the region inside it.

of $S O(2,2)$. Moreover, we would like this orientifolding to also commute with that of BTZ (4). Noting (5), this is only possible if the orientifolding is acting on the $\tilde{r}$ coordinate and not $t$ and $\phi$. Explicitly, that is possible if the orientifold $\mathbb{Z}_{2}$ is $\tilde{r}^{2} \leftrightarrow \tilde{\rho}^{2}$. This $\mathbb{Z}_{2}$ does not have a simple (linear) realization on $X_{i}$ and $T_{i}$ coordinates. In the BTZ coordinate system (6) this orientation changing $\mathbb{Z}_{2}$ is hence

$$
\left(\tau, r^{2}, \varphi\right) \longleftrightarrow\left(\tau, \rho^{2}, \varphi\right)
$$

(Note that $\varphi$ is compact while $\tau$ is not.)

We use ordinary BTZ geometry as the basis for studying the $\mathbb{Z}_{2}$ invariant solution in the covering space. As seen in Fig 2 this $\mathbb{Z}_{2}$ invariant geometry is indeed a double cover of the O-BTZ geometry, i.e. two O-BTZ geometries or two halves of standard BTZ geometries glued at $r^{2}=\rho^{2}=4 G \ell^{2} M$. The metric for the double cover of $\mathrm{O}-\mathrm{BTZ}$ is

$$
\begin{aligned}
d s^{2} & =\left[\rho^{2} \theta(\Phi)+r^{2} \theta(-\Phi)\right] d \tau^{2}-8 G \ell J d \tau d \varphi \\
& +\left[r^{2} \theta(\Phi)+\rho^{2} \theta(-\Phi)\right] d \varphi^{2}+\frac{r^{2} d r^{2}}{16 G^{2} J^{2}-\frac{r^{2} \rho^{2}}{\ell^{2}}}
\end{aligned}
$$

where $\theta(X)$ is the step function and

$$
\Phi=r^{2}-4 G \ell^{2} M=\frac{r^{2}-\rho^{2}}{2} .
$$

In the coordinate system (10) $\tau$ and $\varphi$ are both dimen- 
sionless. The volume element of the geometry is

$$
\begin{aligned}
d V & =\ell d \tau d \varphi(\theta(\Phi) r d r+\theta(-\Phi) \rho d \rho) \\
& =\ell r d \tau d \varphi d r(\theta(\Phi)-\theta(-\Phi)) .
\end{aligned}
$$

That is, as expected and by construction, the two $\mathrm{AdS}_{3}$ regions on the opposite sides of the dashed line in Fig. 2 have opposite orientations.

With the above choice, metric is clearly continuous at $\Phi=0$. Next, one should make sure that the Israel matching conditions 11] at the junction are also met. For the latter we use the formulation developed in [12]. It is straightforward to show that, in notations of [12], the Ricci tensor has the following jump at $\Phi=0$

$$
\breve{R}_{\mu \nu}=64 G^{2}\left(\ell^{2} M^{2}-J^{2}\right) \operatorname{diag}(1,0,-1) \delta(\Phi)
$$

in $(\tau, r, \varphi)$ frame. As we see the jump is caused by a $2 D$ object located at $\Phi=0$ with stress tensor

$$
S_{\mu \nu}=T \sqrt{g_{2}} \operatorname{diag}(1,0,-1),
$$

where $g_{2}=16 G^{2} \ell^{2}\left(\ell^{2} M^{2}-J^{2}\right)$ is the determinant of the two dimensional $\tau \varphi$ part of metric (10) at $\Phi=0$, which is the metric at the junction, and

$$
T=\frac{1}{4 \pi G \ell} .
$$

In the region between two horizons $r$ is the time-like coordinate and hence the orientifold fixed locus is a space-like orientifold with metric of a cylinder, the SOC and $T$ may be associated with the tension of the space-like orientifold cylinder. $^{2}$

Note that the jump in curvature, and hence the stress tensor (13), vanishes for two special cases: massless BTZ $(\ell M=J=0)$ and extremal BTZ $(\ell M=|J|)$. For these two cases the junction is a light-like cylinder (rather than being space-like) and the orientifolding does not need an orientifold string of the type discussed above [15].

As the $\mathbb{Z}_{2}$ (9) and the BTZ orbifolding (5) commute, the O-BTZ solution (10) may be considered as a BTZ solution constructed upon the "O- $\mathrm{AdS}_{3}$ ", the metric for the double cover of which is

$$
d s^{2}=\left(-|\tilde{\Phi}|+\frac{\ell^{2}}{2}\right) d t^{2}-\ell^{2} \frac{d \tilde{r}^{2}}{\tilde{\rho}^{2}}+\left(|\tilde{\Phi}|+\frac{\ell^{2}}{2}\right) d \phi^{2}
$$

in coordinate system (3), where $\tilde{\Phi}=\tilde{r}^{2}-\ell^{2} / 2$. One may perform the matching condition analysis in the covering $\mathrm{AdS}_{3}$ space at $\tilde{\Phi}=0$ and verify that they are satisfied upon insertion of a space-like orientifold plane with tension $T$ given in (14) at $\tilde{\Phi}=0$. The orientifold fixed

\footnotetext{
2 The fact that at the orientifold fixed locus Ricci tensor has a jump is similar to the $R^{2} / \mathbb{Z}_{k}$ orbifold case the analysis of which has been carried out in [13.
}

plane, however, becomes light-like when we approach the conformal boundary of $\mathrm{AdS}_{3} \cdot{ }^{3}$

Remarkably, computing the jump in the value of the density of the gravity action moving from $I I_{-}$to $I I_{+}$ region ( $c f$. Fig 2) one finds

$$
\begin{aligned}
\Delta S & =\frac{1}{16 \pi G} \int d V\left(R+\frac{2}{\ell^{2}}\right)=\frac{1}{\pi} \int d \tau d \varphi \sqrt{\ell^{2} M^{2}-J^{2}} \\
& =\int T \sqrt{g_{2}} d \tau d \varphi .
\end{aligned}
$$

\section{Discussion and outlook}

We have introduced a new class of locally $\mathrm{AdS}_{3}$ solutions to $\mathrm{AdS}_{3}$ Einstein gravity, the O-BTZ black holes. These are BTZ black holes built upon an $\mathrm{AdS}_{3}$ with an orientifold projection $\left(\mathrm{O}-\mathrm{AdS} \mathrm{S}_{3}\right)$ the fixed locus of which is a space-like cylinder. Away from the fixed locus the metric for the O-BTZ is exactly the same as ordinary BTZ and hence they asymptote to $\mathrm{O}-\mathrm{AdS}_{3}$, and are specified by two parameters, ADM mass $M$ and angular momentum $J$. The orientifold projection used is chosen such that it keeps the orientation on (conformal) boundary of $\mathrm{AdS}_{3}$. Having an orientable boundary is important for having a well-defined $2 D$ CFT with a positive definite energy.

As can be seen from the Penrose diagram depicted in Fig,2, 4 O-BTZ geometries do not have the inner horizon and the region behind it. From the viewpoint of an observer outside the horizon O-BTZ geometry is indistinguishable from an ordinary BTZ and in particular its horizon area is $2 \pi r_{+}$, where $r_{+}$can be computed in terms of $M$ and $J$ using (8). Therefore, laws of black hole thermodynamics for our solutions is written exactly in the same way as for the ordinary BTZ.

The Brown-Henneaux analysis [6] only makes use of the asymptotic behavior of diffeomorphisms, that the gravity part of the action is pure $\mathrm{AdS}_{3}$ Einstein gravity, and is independent of the details of the geometry away from the boundary. Therefore, analysis of [6] in a straightforward way generalizes to our case and one finds a Virasoro algebra with central charge (1) for the O-BTZ solutions [15]. This suggests that there is a $\mathrm{CFT}_{2}$ at the central charge (11) and that our O-BTZ solutions are states in this CFT.

Many other aspects of the $\mathrm{O}-\mathrm{AdS}_{3}$ and $\mathrm{O}-\mathrm{BTZ}$ geometries such as geodesic motion in these backgrounds, dis-

\footnotetext{
${ }^{3}$ We comment that $\mathrm{AdS}_{3}$ in Poincaré coordinates, $d s^{2}=$ $\ell^{2}\left[u^{2}\left(-d t^{2}+d x^{2}\right)+\frac{d u^{2}}{u^{2}}\right], u>0$, which appears in the near horizon limit of D1-D5 system [14] can be extended in a similar way to beyond $u^{2}>0$ region by replacing $u^{2}$ with $\left|u^{2}\right|$. In order to make this a solution to $\mathrm{AdS}_{3}$ Einstein gravity we need to add an orientifold cylinder with tension (14) at $u^{2}=0$. Note that $u=0$ is a light-like (rather than space-like) direction.

${ }^{4}$ Note that Fig 2 shows Penrose diagram of O-BTZ geometry in its covering space and that $\mathrm{O}-\mathrm{BTZ}$ is the region limited to two successive dashed lines.
} 
cussion about the possible dual $\mathrm{CFT}_{2}$, extension of proposal made in [16] for the initial (Hartle-Hawking) entangled state to the O-BTZ backgrounds and their relevance for addressing the pure $\mathrm{AdS}_{3}$ Einstein gravity problems (cf. [5]) will be discussed in upcoming publications.

We would like to thank Vijay Balasubramanian, Jan de Boer, Chethan Krishnan, Kumar S. Narain, Joan Simón, Erik Verlinde for comments and especially Kostas Skenderis for discussions. F.L. would like to thank IPM for hospitality during the course of this project. M.M.Sh-J would like to thank the Abdus-Salam ICTP where this work completed.

* Electronic address: loran@cc.iut.ac.ir

$\dagger$ Electronic address: jabbari@theory.ipm.ac.ir

[1] M. Banados, C. Teitelboim and J. Zanelli, "The Black hole in three-dimensional space-time," Phys. Rev. Lett. 69, 1849 (1992). arXiv:hep-th/9204099.

[2] M. Banados, M. Henneaux, C. Teitelboim and J. Zanelli, "Geometry of the $(2+1)$ black hole," Phys. Rev. D 48, 1506 (1993). arXiv:gr-qc/9302012.

[3] A. R. Steif, "The Quantum stress tensor in the threedimensional black hole," Phys. Rev. D 49, 585 (1994). arXiv:gr-qc/9308032.

[4] G. Lifschytz and M. Ortiz, "Scalar field quantization on the (2+1)-dimensional black hole background," Phys. Rev. D 49, 1929 (1994). arXiv:gr-qc/9310008.

[5] E. Witten, "Three-Dimensional Gravity Revisited," arXiv:0706.3359 hep-th]; A. Maloney and E. Witten, "Quantum Gravity Partition Functions in Three Dimensions," arXiv:0712.0155 [hep-th]; A. Strominger, "Five Problems in Quantum Gravity," Nucl. Phys. Proc. Suppl. 192-193, 119 (2009). arXiv:0906.1313 [hep-th]].

[6] J. D. Brown and M. Henneaux, "Central Charges in the Canonical Realization of Asymptotic Symmetries: An Example from Three-Dimensional Gravity," Commun.
Math. Phys. 104, 207 (1986).

[7] P. Kraus, H. Ooguri and S. Shenker, "Inside the horizon with AdS/CFT," Phys. Rev. D 67, 124022 (2003). arXiv:hep-th/0212277.

[8] V. Balasubramanian, T. S. Levi, "Beyond the veil: Inner horizon instability and holography," Phys. Rev. D 70, 106005 (2004). arXiv:hep-th/0405048; C. Krishnan, “ Tomograms of spinning black holes," Phys. Rev. D 80, 126014 (2009). arXiv:0911.0597[hep-th]].

[9] N. Drukker, B. Fiol and J. Simon, "Goedel's universe in a supertube shroud," Phys. Rev. Lett. 91, 231601 (2003). arXiv:hep-th/0306057.

[10] O. Coussaert and M. Henneaux, "Self-dual solutions of $2+1$ Einstein gravity with a negative cosmological constant," arXiv:hep-th/9407181. V. Balasubramanian, A. Naqvi and J. Simon, "A multi-boundary AdS orbifold and DLCQ holography: A universal holographic description of extremal black hole horizons," JHEP 0408, 023 (2004). arXiv:hep-th/0311237.

[11] W. Israel, "Singular hypersurfaces and thin shells in general relativity," Nuovo Cim. B 44S10, 1 (1966) [Erratumibid. B 48, 463 (1967 NUCIA,B44,1.1966)].

[12] R. Mansouri and M. Khorrami, "Equivalence of DarmoisIsrael and Distributional-Methods for Thin Shells in General Relativity," J. Math. Phys. 37, 5672 (1996). arXiv:gr-qc/9608029.

[13] D. V. Fursaev and S. N. Solodukhin, "On The Description Of The Riemannian Geometry In The Presence Of Conical Defects," Phys. Rev. D 52, 2133 (1995) arXiv:hep-th/9501127.

[14] J. M. Maldacena, "The large N limit of superconformal field theories and supergravity," Adv. Theor. Math. Phys. 2, 231 (1998) [Int. J. Theor. Phys. 38, 1113 (1999)]. arXiv:hep-th/9711200.

[15] F. Loran and M.M. Sheikh-Jabbari, "Orientifolded Locally $\mathrm{AdS}_{3}$ Geometries," To appear.

[16] J. M. Maldacena, "Eternal black holes in Anti-de-Sitter," JHEP 0304, 021 (2003). arXiv:hep-th/0106112. 